To appear in the Astrophysical Journal, Letters

\title{
Observations of DG Tauri with the Keck Interferometer
}

M. Colavita ${ }^{1}$, R. Akeson ${ }^{2}$, P. Wizinowich ${ }^{3}$, M. Shao ${ }^{1}$, S. Acton ${ }^{3}$, J. Beletic ${ }^{3}$, J. Bell ${ }^{3}$, J. Berlin ${ }^{1}$,

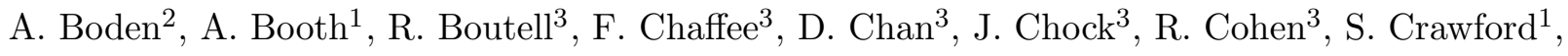
M. Creech-Eakman ${ }^{1}$, G. Eychaner ${ }^{1}$, C. Felizardo ${ }^{2}$, J. Gathright ${ }^{3}$, G. Hardy ${ }^{1}$, H. Henderson ${ }^{2}$, J. Herstein ${ }^{2}$, M. Hess ${ }^{3}$, E. Hovland ${ }^{1}$, M. Hrynevych ${ }^{3}$, R. Johnson ${ }^{1}$, J. Kelley ${ }^{1}$, R. Kendrick ${ }^{3}$, C. Koresko ${ }^{2}$, P. Kurpis ${ }^{3}$, D. Le Mignant ${ }^{3}$, H. Lewis ${ }^{3}$, E. Ligon ${ }^{1}$, W. Lupton ${ }^{3}$, D. McBride ${ }^{3}$,

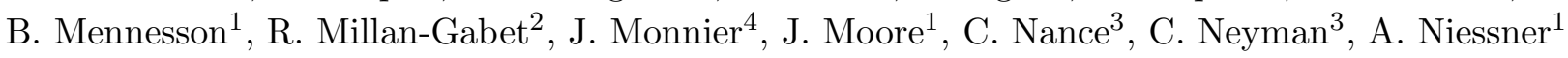
D. Palmer ${ }^{1}$, L. Reder ${ }^{1}$, A. Rudeen ${ }^{3}$, T. Saloga ${ }^{3}$, A. Sargent ${ }^{2}$, E. Serabyn ${ }^{1}$, R. Smythe ${ }^{1}$, P. Stomski ${ }^{3}$, K. Summers ${ }^{3}$, M. Swain ${ }^{1}$, P. Swanson ${ }^{1}$, R. Thompson ${ }^{1}$, K. Tsubota ${ }^{3}$,

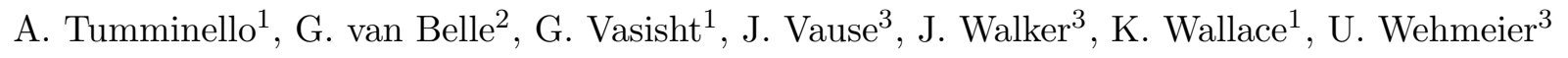

\begin{abstract}
We present the first science results from the Keck Interferometer, a direct-detection infrared interferometer utilizing the two 10-meter Keck telescopes. The instrument and system components are briefly described. We then present observations of the T Tauri object DG Tau, which is resolved by the interferometer. The resolved component has a radius of 0.12 to $0.24 \mathrm{AU}$, depending on the assumed stellar and extended component fluxes and the model geometry used. Possible origins and implications of the resolved emission are discussed.
\end{abstract}

Subject headings: instrumentation: interferometers - stars: pre-main sequence - circumstellar matter

\section{Introduction}

The sensitivity provided by using large aperture telescopes in optical/infrared interferometers will greatly expand the range of astronomical sources which can be studied on milliarcsecond (mas) scales. One such facility is the Keck Interferometer (KI), and we present its first science results: observations of the young stellar object DG Tau.

\footnotetext{
${ }^{1}$ Jet Propulsion Laboratory, 4800 Oak Grove Dr., Pasadena, CA 91109; mcolavit@s383.jpl.nasa.gov

${ }^{2}$ Michelson Science Center, 770 S. Wilson Ave., Pasadena, CA 91125; rla@ipac.caltech.edu

${ }^{3}$ W.M.Keck Observatory, California Association for Research in Astronomy, 65-1120 Mamalahoa Hwy., Kamuela, HI 96743; peterw@keck.hawaii.edu

${ }^{4}$ Univ. of Michigan, 941 Dennison Bldg, Ann Arbor, MI, 48109
} 
The Keck Interferometer ${ }^{5}$ is a NASA-funded joint development among the Jet Propulsion Laboratory (JPL), the California Association for Research in Astronomy, and the Michelson Science Center at the California Institute of Technology ${ }^{6}$, to interferometrically combine the two 10 meter Keck telescopes for high sensitivity near-infrared(IR) visibility amplitude measurements, mid-IR nulling interferometry at $10-12 \mu \mathrm{m}$, and differential-phase interferometry at $1.6-5 \mu \mathrm{m}$. First fringes were obtained in 2001 March using the two Kecks with their adaptive optics (AO) systems, with subsequent activities directed toward improving visibility-mode performance and commencing shared-risk science.

\section{Instrument Description}

Instrument details are given by Colavita \& Wizinowich (2002); in brief, KI uses pupil-plane combination between the two 10-m Keck telescopes, providing an $85-\mathrm{m}$ baseline oriented at $38^{\circ}$ ( $\mathrm{E}$ of $\mathrm{N}$ ). Both telescopes have adaptive optics systems (Wizinowich et al. 2002), which are used for all interferometer observations. For the measurements described here, a single beam from each telescope is routed through the telescope coude train to a beam combining laboratory. Optical path delay is implemented in two stages, with long delay lines (LDLs), which are stationary during an observation, and fast delay lines (FDLs), which track sidereal motion. The available delay on the sky for a given LDL position is $\pm 15 \mathrm{~m}$. The FDLs incorporate measurements from local laser metrology, an end-to-end laser metrology system, and telescope accelerometers for fast servo control and to stabilize the optical path. The KI control system is described by Booth et al. (2002).

Angle tracking to stabilize the images from the individual telescopes is implemented using a HAWAII near-IR array camera system which controls fast tip/tilt mirrors in the beam combining lab. For the data presented here, the angle tracker operated at $J(1.2 \mu \mathrm{m})$ with a $100 \mathrm{~Hz}$ frame rate. Fringe tracking and science measurements use a second HAWAII near-IR array camera system which controls the FDLs (Vasisht et al. 2002). The camera is fed using single-mode fluoride optical fibers from a free-space beam-combination breadboard, and provides a white-light channel from one beamsplitter output and a low-resolution spectrometer channel from the complementary output. The (single-mode) field-of-view on the sky is $\sim 50$ mas (FWHM) at $2.2 \mu \mathrm{m}$. The fringe tracker implements a 4-bin synchronous fringe demodulation algorithm similar to that used at the Palomar Testbed Interferometer (PTI) (Colavita et al. 1999). For the data presented here, the system operated at $K^{\prime}$, with 4-pixel dispersion on the spectrometer side, and used a $500 \mathrm{~Hz}$ frame rate.

\footnotetext{
${ }^{5}$ Additional information at http://planetquest.jpl.nasa.gov/

${ }^{6}$ Additional information at http://msc.caltech.edu/
} 


\section{Results}

\subsection{DG Tau}

$\mathrm{T}$ Tauri stars are pre-main sequence objects with stellar masses less than $\sim 2 \mathrm{M}_{\odot}$ and ages of 1 to 10 million years. The canonical model for these sources comprises a central star surrounded by a circumstellar disk of size 100 AU, a collimated jet or outflow, and perhaps some residual material in a more extended envelope. Due to the high resolution required ( $1 \mathrm{AU}=7$ mas at 140 pc), direct observations of the central region have been limited to date. Characterizing the inner disk properties is important for understanding hydrodynamic disk winds and the initial conditions of planet formation. Given the spatial resolution of current facilities, long-baseline infrared interferometry provides an ideal method for observing these inner regions, which are traced by material thermally emitting in the near-IR.

Previous interferometric observations have resolved several types of young stellar objects (YSO): FU Ori (Malbet et al. 1998), T Tauri (Akeson et al. 2000), and Herbig AeBe sources

(Millan-Gabet et al. 1999; Millan-Gabet, Schloerb, \& Traub 2001). For the T Tauri and Herbig objects, the measured visibilities are generally not consistent with a geometrically flat disk with an inner radius less than a few stellar radii as predicted by spectral energy distribution fitting. Tuthill, Monnier, \& Danchi (2001) and Natta et al. (2001) proposed that the inner radius is located at the destruction radius for directly heated dust, which can resolve this discrepancy. However, due to the sensitivity limits of the facilities used, the YSOs observed to date, particularly the T Tauris, are the brightest infrared examples and may not be representative.

Here we present observations of DG Tau ( $d=140$ pc), a T Tauri star with a more typical luminosity, which has a well-studied jet (see e.g. Lavalley-Fouquet, Cabrit, \& Dougados (2000); Bacciotti et al. (2002) and references therein) and a circumstellar disk observed in both dust continuum and molecular gas (Kitamura, Kawabe, \& Saito 1996a,b; Testi et al. 2002). The infrared spectral index for DG Tau is roughly flat, placing it in the class II evolutionary category (Lada 1987). DG Tau has a $K$ magnitude of $6.98 \pm 0.013$ (2MASS, 1997 November 30) and an average $V$ magnitude of 12.43 (Kenyon \& Hartmann 1995). The optical jet has a position angle (PA) of $226^{\circ}$ and a derived inclination of $38^{\circ}$ from the line of sight (Bacciotti et al. 2002). On larger scales, the disk inclination has been estimated as $70^{\circ}$ at $\sim 100 \mathrm{AU}$ and at $40^{\circ}$ at $\sim 2800 \mathrm{AU}$ (Kitamura, Kawabe, \& Saito 1996a,b).

\subsection{Observations and data reduction}

DG Tau was observed with the Keck Interferometer on 2002 October 23 and 2003 February 13 UT. The data presented here are from the white-light channel $\left(\lambda_{\text {center }}=2.18 \mu \mathrm{m}\right.$ and $\left.\Delta \lambda \sim 0.3 \mu \mathrm{m}\right)$. Observations consisted of a series of interleaved integrations on the source and several calibrators. Each integration includes 120 seconds of fringe data followed by measurements of the background 
flux, the individual fluxes in the two arms of the interferometer, and the foreground flux. The data presented are the visibility amplitude squared, an unbiased quantity, normalized such that an unresolved object has $\mathrm{V}^{2}=1.0$.

The system visibility $\left(\mathrm{V}_{\text {sys }}^{2}\right)$, i.e., the instrumental response to a point source, is measured with respect to calibrator stars HD 282230, HD 283668, and HD 29050. The calibrators are located 5.3 $1.7^{\circ}$ and $3.6^{\circ}$ from DG Tau, respectively, and have $K$ magnitudes of 7.3, 7.0 and 7.1, well matched to DG Tau's $K$ magnitude. The calibrator angular sizes were derived by fitting photometry from SIMBAD and 2MASS. All calibrators have angular diameters $<0.3$ mas, and are unresolved by the interferometer. Source and calibrator data were corrected for detection biases as described by Colavita (1999) and averaged into 5 second blocks. $\mathrm{V}_{\text {sys }}^{2}$ is calculated for each DG Tau data point using an average of the calibrator measurements weighted by the internal scatter in the calibrator data and the temporal and angular proximity to the target data point (Boden et al. 1998). In

addition, the $\mathrm{V}_{\text {sys }}^{2}$ error includes the uncertainty in the calibrator size, assumed to be 0.1 mas. The calibrated data points for the target source are the average of the 5 sec blocks in each integration, with an uncertainty given by the quadrature of the internal scatter and the uncertainty in $\mathrm{V}_{\text {sys }}^{2}$. The average $\mathrm{V}^{2}$ for DG Tau is 0.37 with a measurement uncertainty of 0.02 .

Several checks were performed to verify the measured visibility. The calibrator $K$ magnitudes are within 0.3 magnitudes of DG Tau, minimizing any errors due to flux dependencies in the fringe tracker. For comparison to the white light data presented, a synthetic wide-band measurement was constructed by summing the four spectrometer channels. These data are within $1 \sigma$ of the white-light data. In addition to measurement errors, there may be systematic errors in $\mathrm{V}^{2}$. These have been estimated using observations of binary systems with known orbital parameters. These binary systems had $\mathrm{V}^{2}$ of 0.2 to 0.4 , similar to the DG Tau visibility, and an rms from the predicted visibility of 0.05 . Therefore, as a conservative estimate of the KI performance, we assign a systematic error of 0.05 to the DG Tau data and quadratically sum this error with the measurement uncertainties (Fig. 1). The systematic error dominates the measurement error, resulting in an average $\mathrm{V}^{2}$ for DG Tau of $0.37 \pm 0.05$ at a projected baseline length of 84.7 meters.

\subsection{Models of source structure}

As seen in Fig. 1, DG Tau is clearly resolved by KI. We have used simple geometric models of the source brightness to estimate the size scale of the infrared emission region. Several components may be contributing to the measured visibility: the stellar photosphere, which is unresolved in our observations; emission from compact material, which is less than the 5 mas fringe spacing and is partially resolved; and extended emission, which is completely resolved and contributes incoherently.

Before the size of the compact component can be determined, the stellar and incoherent contributions must be quantified. In the spectrum of DG Tau, the line features are veiled by continuum 


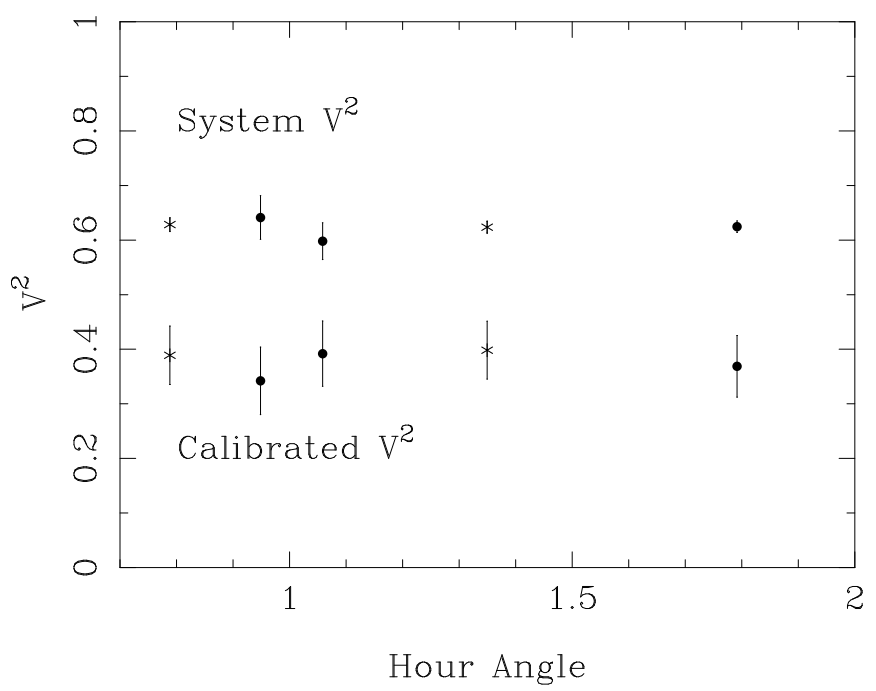

Fig. 1. - The calibrated DG Tau visibilities (bottom points) and system visibilities (top points). Data from 2002 October are labeled with stars and from 2003 February with circles. The projected baseline ranges from 84.2 to 84.9 meters at PA $32^{\circ}$ to $25^{\circ}$ (E of N). The DG Tau error bars include both the measurement and systematic components.

emission, making the spectral type difficult to determine, with a range in the literature of K7 to M0 (Kenyon \& Hartmann 1995). We have used two methods to calculate the stellar contribution to the $K$ flux: 1$)$ using the effective temperature $\left(\mathrm{T}_{\text {eff }}=3890 \mathrm{~K}\right)$ and extinction $\left(\mathrm{A}_{\mathrm{V}}=1.6\right)$ from Beckwith et al. (1990) and fitting the de-reddened optical photometry, which gives a stellar/total flux ratio of 0.21 ; and 2) simply adopting the flux ratio of 0.41 derived by Johns-Krull \& Valenti (2001) using infrared spectroscopy. To estimate any extended component, we use the lunar occultation observations of Leinert et al. (1991) and Chen et al. (1992). These measurements are sensitive to emission on scales from 5 mas to $1^{\prime \prime}$. Leinert et al. (1991) fit the extended component at $K$ with a 45 mas (FWHM) Gaussian with a flux ratio (extended/total) of 0.23. Chen et al. (1992) derived similar results from independent observations. Leinert et al. (1991) also used speckle interferometry, which revealed a 0 "'85 symmetric envelope. As this larger envelope contributes only $3 \%$ of the $K$ flux, we do not include it in our modeling. Based on the flux and size of the 45 mas emission (hereafter, the extended component), Leinert et al. (1991) suggest that this emission is likely due to scattered light rather than thermal emission.

We chose two simple geometries to estimate the size of the compact component. Given the observed non-variation of the visibilities with baseline orientation within the limited hour angle range of our observations, we cannot constrain any asymmetry in the source, and therefore consider only symmetric models - a uniform disk and a uniform brightness ring (Table 1). The ring width is set for a given inner diameter by requiring that the ring flux for a typical dust destruction temperature $(1500 \mathrm{~K})$ match the observed $K$ excess for the compact component. Both the stellar (unresolved) and extended (completely resolved) components are included in the modeling. 


\begin{tabular}{lll}
\hline $\mathrm{F}_{\text {star }} / \mathrm{F}_{\text {total }}$ & 0.41 & 0.21 \\
\hline $\mathrm{UD}$ model & & \\
$\quad$ radius (mas) & $1.71 \pm 0.26$ & $1.33 \pm 0.19$ \\
$\quad$ radius (AU) & $0.24 \pm 0.036$ & $0.19 \pm 0.027$ \\
Ring model & & \\
$\quad$ inner radius (mas) & $1.16 \pm 0.17$ & $0.89 \pm 0.14$ \\
$\quad$ inner radius (AU) & $0.16 \pm 0.024$ & $0.12 \pm 0.020$ \\
$\quad$ width (AU) & 0.006 & 0.012 \\
\hline
\end{tabular}

Table 1: The fit radii and uncertainties (including measurement and systematic errors) for the uniform disk (UD) and ring models for two values of the stellar fractional flux (§3.3). All models include an incoherent component with a fractional flux of 0.23 . The ring model width is set as described in the text for a blackbody temperature of $1500 \mathrm{~K}$.

\section{Discussion}

We have estimated the radius of the compact emission region of DG Tau to be 0.12 to $0.24 \mathrm{AU}$ given our estimates for the relative flux of the stellar and extended components. Here we discuss the hypothesis that this resolved component represents thermal emission from the inner regions of the circumstellar disk. Using a standard $\mathrm{T}$ Tauri disk model with a temperature power-law $T \propto r^{-q}, \mathrm{~T}(1 \mathrm{AU})=150 \mathrm{~K}$ and $\mathrm{q}=0.5$ (see e.g. Beckwith et al. (1990)), the $2 \mu \mathrm{m}$ emission arises from material located within 5 mas of the star.

The radii in Table 1 are similar those derived for the disks around the T Tauri stars T Tau N and SU Aur by Akeson et al. (2000), and all are larger than predicted by models based on spectral energy distribution fitting. We use the ring morphology as a simple representation of models where the inner ring radius is set by the dust destruction radius (Dullemond, Dominik, \& Natta 2001; Monnier \& Millan-Gabet 2002), where $R_{\text {dust }}=1 / 2\left(L_{\star} / 4 \pi \sigma T_{\text {dust }}^{4}\right)^{1 / 2}$. Figure 2 shows the radius corresponding to a dust destruction temperature $\left(\mathrm{T}_{\text {dust }}\right)$ of $1500 \mathrm{~K}$ plotted against the fit ring radius for DG Tau. In addition, four T Tauri sources observed at PTI are also plotted (Akeson, Ciardi, \& van Belle 2002). For DG Tau the observed radius is larger than $R_{\text {dust }}$ for $\mathrm{T}_{\text {dust }}=1500 \mathrm{~K}$, while for the PTI sources the observed radius is similar or smaller. Although there are too few sources to generalize, DG Tau is the faintest infrared source of the group.

We now briefly mention several issues and caveats in our determination of the size of the $K$ emission region for DG Tau.

Source variability: DG Tau is known to vary at optical and infrared wavelengths; Skrutskie et al. (1996) measured the $K$ variability to be 0.8 mag. If this variability changes the relative contributions of the stellar, compact and extended components, then the derived size is directly affected. For example, if the scattered light emission at $K$ were 0.39 times the total flux (1.7 times higher than measured by Leinert et al. (1991)), the measured visibility would be accounted for by 


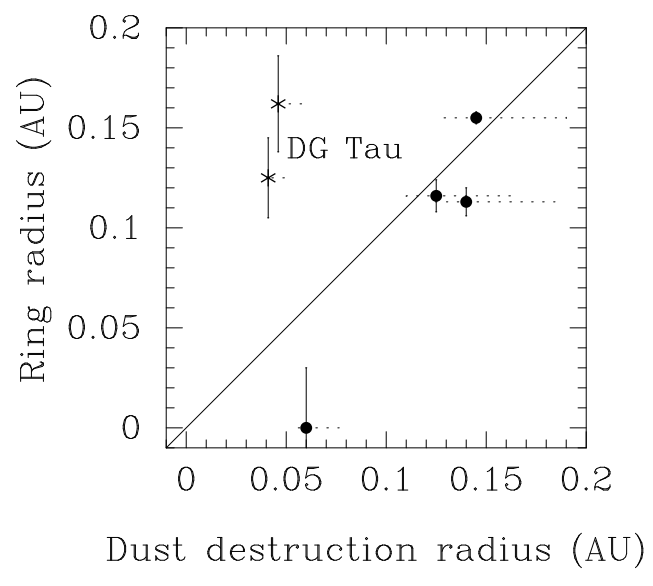

Fig. 2. - A comparison of the dust destruction radius (for $\mathrm{T}_{\text {dust }}=1500 \mathrm{~K}$ ) and the best fit ring model (inner radius) for DG Tau (stars) and four other T Tauri sources observed with PTI (circles). The solid line illustrates the location where these radii are equal. The two DG Tau points correspond to the two stellar models used. The horizontal dotted lines represent a typical range of $\mathrm{T}_{\text {dust }}(1200$ to $1800 \mathrm{~K}$ ). The $\mathrm{y}$-axis error bars do not include the uncertainty in the fractional flux from the unresolved and extended components.

just the stellar (unresolved) and extended components. Although this level of variability is seen in the total $K$ flux, the ratio of scattered to stellar flux should be constant for a brightening of the star.

Extended flux contribution: We have assumed that the 45 mas extended component is circularly symmetric and completely coupled into the fringe tracker. The exact coupling of this emission into the fringe tracker field-of-view is dependent on the extended component shape and on the AO and system performance. The lunar occultation observations of Leinert et al. (1991) and Chen et al. (1992), taken at PAs of $90^{\circ}, 233^{\circ}$ and $256^{\circ}$, resulted in similar size determinations, suggesting this component is roughly symmetric. Any reduction in the extended component coupling would result in a larger size for the partially resolved component. If there were no extended component, we would obtain a ring radii of 0.17 to $0.21 \mathrm{AU}$ (for the two stellar models). Scattered light may be affecting the stellar properties derived by Beckwith et al. (1990) and Johns-Krull \& Valenti (2001). A smaller stellar (unresolved) component would result in a smaller ring radius.

Inclination: For an inclined disk, a less simplistic representation is necessary as the Natta et al. (2001) models are vertically extended at the inner edge. However, in their T Tauri models, the height is much less than the radius at the inner edge, and therefore the measured size will underestimate the physical size. If the inner disk is orthogonal to the jet (which implies a disk PA of $136^{\circ}$ ), the actual size will be only a few percent larger than our measured size given the measurement PA. However, if the DG Tau disk is near edge-on, scattered light in the polar regions could be more significant, increasing the measured size. 
Dust destruction radius: The dust destruction radius depends on the assumed stellar luminosity and on $\mathrm{T}_{\text {dust }}$. For the dust destruction radius to match the measured ring radius at $\mathrm{T}_{\text {dust }}=1500 \mathrm{~K}$, a luminosity of 3 to $5 \mathrm{~L}_{\odot}$ would be required, while the values for the assumed stellar models are $1.7 \mathrm{~L} \odot$ (Beckwith et al. 1990) and $1.4 \mathrm{~L} \odot$ (Johns-Krull \& Valenti 2001). Similarly, for the luminosity values used, a dust destruction temperature of $\mathrm{T}_{\text {dust }}=1100$ to $1300 \mathrm{~K}$ would be required to match the measured ring radius. As discussed by Monnier \& Millan-Gabet (2002), small grains have a larger dust destruction radius.

Multiplicity: An additional point source within the KI field-of-view (50 mas) could account for the measured visibility. Leinert et al. (1991) observed DG Tau with speckle interferometry and found no companion source. Although we see no evidence for visibility variability, as would occur for a coherently contributing source, the presence of a companion can not be completely ruled out from the present KI data.

If our model of the extent and brightness of the DG Tau infrared emission components is correct, the difference between the estimated ring radius and the radius at which $\mathrm{T}_{\text {dust }}=1500 \mathrm{~K}$ is significant. The $\mathrm{V}^{2}$ for DG Tau is $0.37 \pm 0.05$, while the stellar properties of DG Tau predict a $\mathrm{V}^{2}$ of 0.54 to 0.57 (including the extended component) for $\mathrm{T}_{\text {dust }}=1500 \mathrm{~K}$. Although there may be optically thin gas or dust within the derived ring radius, a large gap between the star and the optically thick inner edge of the disk has implications for some hydrodynamic wind models and planet migration theories.

We have demonstrated the basic capabilities of the KI in $\mathrm{V}^{2}$ mode, using the interferometric combination of two AO-equipped 10 meter class telescopes. We present the first KI science result: the detection of clearly resolved near-IR emission from DG Tau, the faintest T Tauri object measured to date with an optical/IR interferometer. These observations provide constraints on theories of circumstellar disks and demonstrate the potential of large aperture optical/IR interferometry in providing information on milliarcsecond scales not only for young stellar objects, but for many areas of stellar and extra-galactic astrophysics.

KI is funded by the National Aeronautics and Space Administration(NASA). Part of this work was performed at the Jet Propulsion Laboratory, California Institute of Technology(Caltech) and at the Michelson Science Center(MSC), under contract with NASA. Observations were obtained at the W.M. Keck Observatory, operated as a scientific partnership among Caltech, the University of California and NASA. The Observatory was made possible by the generous financial support of the W.M. Keck Foundation. The authors wish to recognize and acknowledge the very significant cultural role and reverence that the summit of Mauna Kea has always had within the indigenous Hawaiian community. We are most fortunate to have the opportunity to conduct observations from this mountain. This work has used software from the MSC; the SIMBAD database, operated at CDS, Strasbourg, France; and the NASA/IPAC Infrared Science Archive, operated by JPL under contract with NASA. 


\section{REFERENCES}

Akeson, R. L., Ciardi, D. R., van Belle, G. T., Creech-Eakman, M. J., \& Lada, E. A. 2000, ApJ, 543,313

Akeson, R. L., Ciardi, D. R., \& van Belle, G. T. 2002, Proc. SPIE, 4838, 1037

Bacciotti, F., Ray, T. P., Mundt, R., Eislöffel, J., \& Solf, J. 2002, ApJ, 576, 222

Beckwith, S. V. W., Sargent, A. I., Chini, R. S., \& Guesten, R. 1990, AJ, 99, 924

Boden, A. F., Colavita, M. M., van Belle, G. T., \& Shao, M. 1998, Proc. SPIE, 3350, 872

Booth, A.J. et al., 2002, Proc. SPIE, 4848, 107

Chen, W. P., Howell, R. R., Simon, M., \& Benson, J. A. 1992, ApJ, 387, L43

Colavita, M. M. \& Wizinowich, P. L. 2002, Proc. SPIE, 4838, 79

Colavita, M. M. 1999, PASP, 111, 111

Colavita, M. M. et al. 1999, ApJ, 510, 505

Dullemond, C. P., Dominik, C., \& Natta, A. 2001, ApJ, 560, 957

Johns-Krull, C. M. \& Valenti, J. A. 2001, ApJ, 561, 1060

Kenyon, S. J. \& Hartmann, L. 1995, ApJS, 101, 117

Kitamura, Y., Kawabe, R., \& Saito, M. 1996, ApJ, 457, 277

Kitamura, Y., Kawabe, R., \& Saito, M. 1996, ApJ, 465, 137

Lada, C. J. 1987, IAU Symp. 115: Star Forming Regions, 115, 1

Lavalley-Fouquet, C., Cabrit, S., \& Dougados, C. 2000, A\&A, 356, L41

Leinert, C., Haas, M., Mundt, R., Richichi, A., \& Zinnecker, H. 1991, A\&A, 250, 407

Malbet, F. et al. 1998, ApJ, 507, L149

Millan-Gabet, R., Schloerb, F. P., Traub, W. A., Malbet, F., Berger, J. P., \& Bregman, J. D. 1999, ApJ, 513, L131

Millan-Gabet, R., Schloerb, F. P., \& Traub, W. A. 2001, ApJ, 546, 358

Monnier, J. D. \& Millan-Gabet, R. 2002, ApJ, 579, 694

Natta, A., Prusti, T., Neri, R., Wooden, D., Grinin, V. P., \& Mannings, V. 2001, A\&A, 371, 186 
Skrutskie, M. F., Meyer, M. R., Whalen, D., \& Hamilton, C. 1996, AJ, 112, 2168

Testi, L., Bacciotti, F., Sargent, A. I., Ray, T. P., \& Eislöffel, J. 2002, A\&A, 394, L31

Tuthill, P. G., Monnier, J. D., \& Danchi, W. C. 2001, Nature, 409, 1012

Vasisht, G., Booth, A.J., Colavita, M.M., Johnson, R.L., Ligon, E.R., Morre, J.D., \& Palmer, D.L., 2002, Proc. SPIE, 4838, 824

Wizinowich, P., Le Mignant, D., Stomski, P., Acton, D.S., Contos, A., \& Neyman, C., 2002, Proc. SPIE, 4839, 9 